\title{
ICTIOFAUNA DO RIO ITAJAÍ AÇU, SANTA CATARINA, BRASIL
}

\author{
HOSTIM-SILVA, M. ${ }^{1}$; VICENTE, M.J.D. ${ }^{2}$; FIGNA, V. ${ }^{1}$ \& J.P. ANDRADE ${ }^{2}$ \\ 1 CTTMar, Universidade do Vale do Itajaí, Rua Uruguai, 458 Cx.360 CEP. 88302 - 202 \\ Itajaí-SC, Brasil. \\ ${ }^{2}$ Departamento de Ictiologia da Universidade do Algarve, Campus de Gambelas, 8000 \\ Faro, Portugal.
}

\section{RESUMO}

\begin{abstract}
Este trabalho foi realizado na área estuarina do Rio Itajaí-Açu, Santa Catarina, Brasil (26을 $52^{\prime}-26^{\circ} 55^{\prime} \mathrm{S}$ e $48^{\circ} 37^{\prime}-48^{\circ} 41^{\prime}$ W), no período de setembro de 1995 a agosto de 1996 . As amostragens foram realizadas mensalmente, utilizando-se redes de espera, rede de arrasto e tarrafas, em 4 áreas de coletas. Foram capturados um total de 2217 exemplares, identificados em 18 famílias e 38 espécies de Teleostei.
\end{abstract}

Palavras chaves: ictiofauna; estuário; bioecologia

\section{ICHTHYOFAUNA OF THE ITAJAÍ-AÇU RIVER, SANTA CATARINA, BRAZIL}

\begin{abstract}
This paper aimed to characterize the ichthyofauna in the estuary of the Itajaí-Açú river, Santa Catarina, Brazil (26 $52^{\prime}-26^{\circ} 5^{\prime} \mathrm{S}$ and $48^{\circ} 37^{\prime}-48^{\circ}$ 41 $^{\prime} \mathrm{W}$ ) where four sampling stations were established, from September 1995 to August 1996. Fish were caught with drop nets, a 3 sided beach seine and a bottom net. Total of 2217 individuals belonging to 18 families were captured and 38 Teleostei species identified.
\end{abstract}

Key words: ichthyofauna; estuary; bioecology

\section{INTRODUÇÃO}

A estratégia de estudo das comunidades ictiológicas estuarinas comporta, numa primeira fase, o levantamento qualitativo e/ou quantitativo das espécies e, numa segunda fase, a compreensão das relações ecológicas entre elas (Barnes, 1980; Yáñez-Arancibia et al, 1983). A primeira fase é muito importante, constituindo freqüentemente a base de estudo para a segunda fase, que se revela com interesse científico muito atual e que tem como objetivo a identificação das unidades ecológi- cas homogêneas e das múltiplas interações que se estabelecem ou são estabelecidas entre estas unidades e entre elas e o meio ambiente (Daget, 1976; Amanieu \& Lasserre, 1982). Através da determinação de alterações no quadro faunístico, observáveis a partir de levantamentos periódicos, pode-se detectar se um ambiente se vem modificando em função de impactos ambientais (Moyele \& Cech, 1988).

Os estuários, como fronteira entre os meios marinho, terrestre e fluvial, têm sido considerados dos habitats naturais mais produtivos do mundo, representando áreas vitais de 
alimentação para muitas espécies de aves e de reprodução de peixes, assim como locais tradicionais de pesca costeira. As características dos estuários como ecossistemas muito dinâmicos determinam que o seu estudo assuma enorme interesse para a compreensão dos processos adaptativos de plantas e animais sujeitos a condições ambientais muito variáveis à escala espacial e temporal. Devido ao seu papel em termos de atividades humanas, a maioria dos estuários encontra-se sob grande pressão ambiental (Potter et al.,1990).

Estes ambientes estão bem representados no Estado de Santa Catarina, porém poucas informações foram produzidas sobre as espécies de peixes que vivem nestas áreas. Este trabalho, apresenta informações preliminares sobre a ictiofauna que ocorre na Foz do Rio Itajaí Açu.

\section{METODOLOGIA}

O presente trabalho foi realizado na foz do Rio Itajaí-Açú, Santa Catarina, Brasil (26 $52^{\prime}$ e $26^{\circ} 55^{\prime} \mathrm{S}$ e $48^{\circ} 37^{\prime}$ e $48^{\circ} 41^{\prime} \mathrm{W}$ ), através de coletas mensais, com duração média de 6 horas, no período de setembro de 1995 a agosto de 1996, perfazendo um total de 12 campanhas. As amostragens efetuaram-se sempre da estação mais a montante para a mais jusante, acompanhando a vazante (Figura 01).

Os peixes foram capturados com arrasto de portas, tarrafa e rede de emalhar. A rede de arrasto com $12 \mathrm{~mm}$ de malha (nós opostos) foi utilizada com um esforço de 15 min em cada área; a tarrafa apresentava $20 \mathrm{~mm}$ de malha (nós opostos) e foi utilizada com um esforço de pesca de 3 lances por área e a rede de deriva do tipo feiticeira, constituída por 3 panos, uma central de $80 \mathrm{~mm}$ e dois laterais de $120 \mathrm{~mm}$ (nós opostos), foi utilizada com um esforço de pesca de 2 lances de 15 min cada, para cada área.

O material amostrado foi colocado em sacos de plástico, etiquetado e acondicionado em caixas de isopor contendo gelo, sendo pos- teriormente transportado para o laboratório. A identificação das espécies foi efetuada com base em: Figueiredo \& Menezes (1978); Fisher (1978); Figueiredo \& Menezes (1980); Menezes \& Figueiredo (1980); Menezes \& Figueiredo (1985); Barletta \& Corrêa (1992); Nelson (1994).

De cada exemplar foi registrada a biometria, nomeadamente o comprimento total (CT) e o comprimento padrão (CP), medidos com um ictiômetro de $1 \mathrm{~mm}$ de precisão.

Os dados foram apresentados em forma de tabelas, contendo a lista de espécies distribuídas por família, seguindo o proposto por Nelson (1994). Nesta tabela, apresentou-se, para cada espécie, a abundância numérica, as amplitudes de comprimento total e categoria de freqüência de ocorrência durante o ciclo anual, considerando:

- Espécies muito frequentes: as presentes em mais de $75 \%$ das amostragens;

- Espécies frequentes: as presentes em 50$75 \%$ dos levantamentos;

- Espécies pouco frequentes: as presentes em $25-42 \%$ das amostragens e

- Espécies ocasionais: aquelas que se fizeram representar de $8-17 \%$ dos levantamentos.

\section{RESULTADOS}

Durante o período de amostragem foram capturados 2217 exemplares, totalizando uma biomassa de $101,5 \mathrm{Kg}$, os quais foram identificados em 38 espécies pertencentes a 18 famílias de Teleostei (Tabela I).

Das espécies capturadas na Foz do Rio Itajaí-Açú, Genidens genidens (N=612); Stellifer rastrifer $(\mathrm{N}=605)$ e Micropogonias furnieri $(\mathrm{N}=247)$, foram as que mais se destacaram em número de exemplares para a área amostrada. Entre as espécies menos abundantes para o ambiente estudado, podemos enumerar Sphoeroides testudineus, Awaous tajasica, 


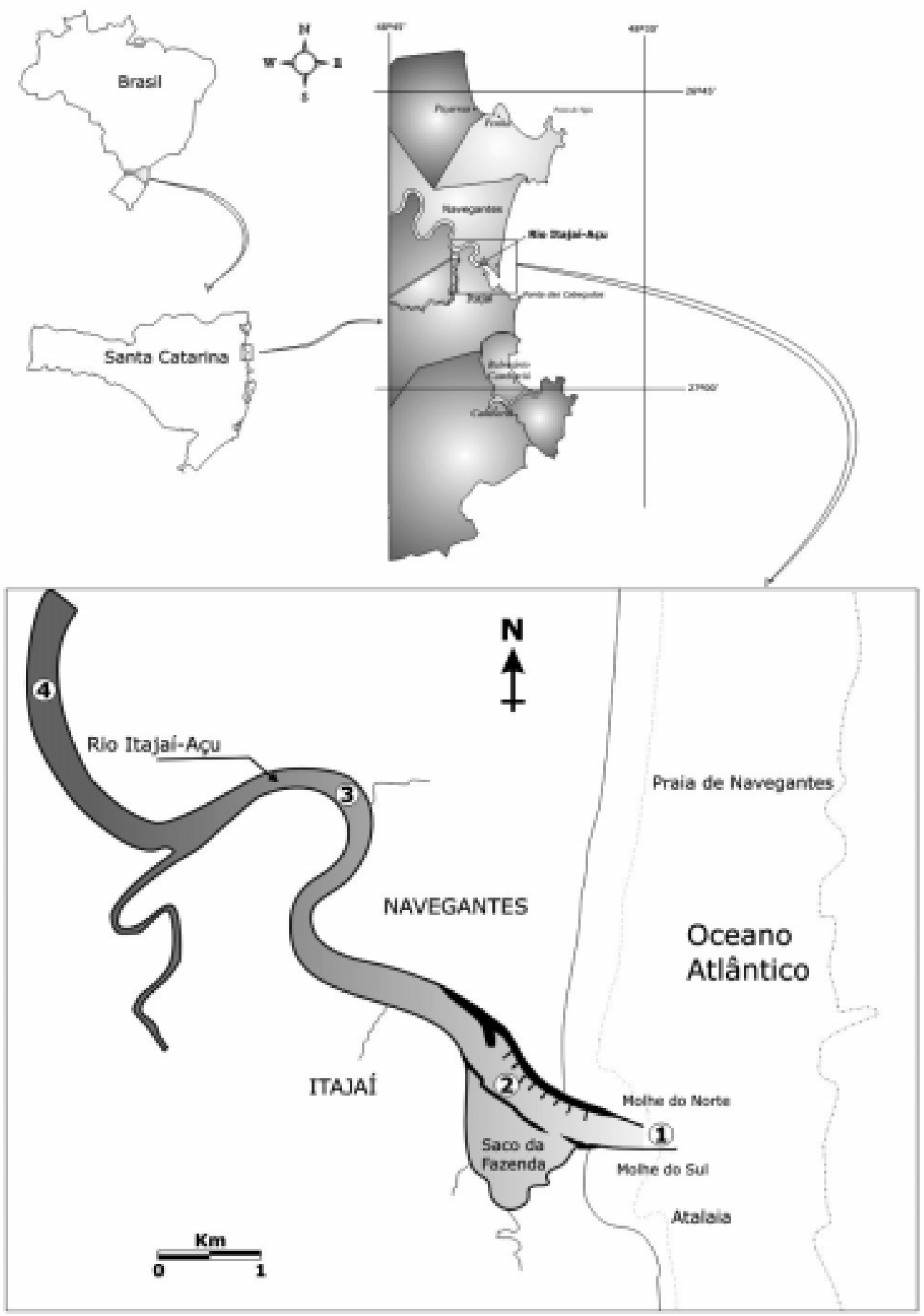

Figura 1 - Localização da Foz do Rio Itajaí-Açu e as estações de amostragem. 
HOSTIM-SILVA, M. et al:: Peixes do rio Itajaí-açu.

Tabela 1. Lista sistemática das espécies capturadas na foz do Rio Itajaí-Açú, segundo NELSON (1994).

\begin{tabular}{|c|c|}
\hline Famílias & Espécies \\
\hline \multirow[t]{3}{*}{ Engraulidae } & Anchoa spinifer (Valenciennes, 1848) \\
\hline & Lycengraulis grossidens (Agassiz, 1829) \\
\hline & Cetengraulis edentulus (Cuvier, 1829) \\
\hline Clupeidae & Harengula clupeola (Cuvier, 1829) \\
\hline \multirow[t]{3}{*}{ Ariidae } & Cathorops spixii (Agassiz, 1829) \\
\hline & Genidens genidens (Valenciennes, 1839) \\
\hline & * Netuma barba (Lacépède, 1803) \\
\hline Pimelodidae & Pimelodus maculatus Lacépède, 1803 \\
\hline Mugilidae & Mugil curema Valenciennes, 1836 \\
\hline Centropomidae & Centropomus parallelus Poey, 1860 \\
\hline \multirow[t]{3}{*}{ Carangidae } & Selene setapinnis (Mitchill, 1815) \\
\hline & Selene vomer (Linnaeus, 1758) \\
\hline & Trachinotus falcatus (Linnaeus, 1758) \\
\hline \multirow[t]{4}{*}{ Gerreidae } & Diapterus rhombeus (Cuvier, 1829) \\
\hline & Eucinostomus melanopterus (Bleeker, 1863) \\
\hline & Eucinostomus gula (Quoy \& Gaimard, 1824) \\
\hline & Eugerres brasilianus (Cuvier, 1830) \\
\hline Haemulidae & Pomadasys corvinaeformis (Steindachner, 1868) \\
\hline \multirow[t]{8}{*}{ Sciaenidae } & Bairdiella ronchus (Cuvier, 1830) \\
\hline & Cynoscion leiarchus (Cuvier, 1830) \\
\hline & Isopisthus parvipinnis (Cuvier, 1830) \\
\hline & Micropogonias furnieri (Desmarest, 1823) \\
\hline & Ophioscion punctatissimus Meek \& Hildebrand, 1925 \\
\hline & Paralonchurus brasiliensis (Steindachner, 1875) \\
\hline & Stellifer brasiliensis (Schultz, 1945) \\
\hline & Stellifer rastrifer (Jordan, 1889) \\
\hline \multirow[t]{4}{*}{ Gobiidae } & Awaous tajasica (Lichtenstein, 1822) \\
\hline & Bathygobius soporator (Valenciennes, 1837) \\
\hline & Gobioides broussonneti Lacépède, 1800 \\
\hline & Gobionellus oceanicus (Pallas, 1770) \\
\hline Ephippidae & Chaetodipterus faber (Broussonet, 1782) \\
\hline Trichiuridae & Trichiurus lepturus Linnaeus, 1758 \\
\hline Achiridae & Catathyridium garmani (Jordan, 1889) \\
\hline Paralichthyidae & Citharichthys spilopterus Günther, 1862 \\
\hline Cynoglossidae & Symphurus tessellatus (Quoy \& Gaimard, 1824) \\
\hline \multirow[t]{2}{*}{ Tetraodontidae } & Sphoeroides testudineus (Linnaeus, 1758) \\
\hline & Lagocephalus laevigatus (Linnaeus, 1766) \\
\hline Diodontidae & Cyclichthys spinosus (Linnaeus, 1758) \\
\hline
\end{tabular}

* Genidens barbus (Lacépède, 1803) por Marceniuk, A.P. \& Menezes, N.A. 2002. Ariidae . In: Buckup, P.A. \& Menezes, N.A. (eds.) Catálogo dos Peixes Marinhos e de Água Doce do Brasil. URL: http://www.mnrj.ufrj.br/ catalogo/

Cynoscion leiarchus, Isopisthus parvipinnis, Eugerres brasilianus e Centropomus parallelus que se fizeram representar por exemplares únicos ao longo dos 12 meses amostrados.

A família Sciaenidae, foi a que apresentou maior riqueza por família com 8 espécies, seguida por Gobiidae com 4. Já Ariidae, Carangidae e Gerreidae, foram representadas por 3 espécies. Entre as famílias que apresentaram menor riqueza específica podemos citar as famílias Clupeidae, Pimelodidae, Mugilidae, Centropomidae, Haemulidae, Ephippidae, 
Trichiuridae, Achiridae, Paralichthyidae, Cynoglossidae e Diodontidae que apenas se fizeram representar por uma espécie durante todo o ciclo anual.

Dentre as espécies referidas, $G$. genidens mostrou ser a mais abundante, constando em todas as áreas de amostragem. As espécies de interesse comercial, S. rastrifer e M. furnieri foram as que mais se destacaram dentro da família Sciaenidae, ocorrendo em maiores quantidades na área próxima ao mar e em todas as estações do ano.

Quanto à permanência na área de estudo, podemos ressaltar o elevado número de exemplares presentes na estação mais perto do mar $(\mathrm{N}=1087)$ e enumerar as espécies Lycengraulis grossidens, Eucinostomus gula, Cetengraulis edentulus, Harengula clupeola, Mugil curema, Eugerres brasilianus, Pomadasys corvinaeformis, Cynoscion leiarchus, Isopisthus parvipinnis, Ophioscion punctatissimus e Trichiurus lepturus, que apenas marcaram presença na estação 1 .

Outras, como é o caso das espécies Pimelodus maculatus, Selene vomere Selene setapinnis, apenas ocorreram na estação mais a montante.

Em relação às espécies com maior freqüência de ocorrência no período de amostragens, pode-se destacar por ordem decrescente as espécies Micropogonias furnieri, Symphurus tessellatus, Genidens genidens, Citharichthys spilopterus e Stellifer rastriferque ocorreram em todas as estações de amostragem, apresentando (com exceção da espécie $S$. tessellatus) maiores abundâncias nas áreas perto do mar. Ressalta-se que a maior percentagem da ictiofauna amostrada foi constituída por espécies ocasionais (55\%).

\section{DISCUSSÃO}

A composição ictiofaunística da foz do Rio Itajaí-Açu é menor do que encontrada em outros estudos semelhantes realizados na costa sul do Brasil, nos quais se verificou um nú- mero bastante alto de espécies (Chao et al., 1982; Chaves \& Vendel, 2001). A presença nas capturas de exemplares das famílias Gerreidae e Carangidae no rio Itajaí-Açú, reforça as conclusões dos levantamentos de ictiofauna realizados anteriormente em outros ambientes estuarinos, tais como o manguezal de Itacorubi (Soriano-Sierra \& Sierra de Ledo, 1998) e o manguezal do Rio Camboriú (Rodrigues et al., 1994). A ocorrência das famílias Sciaenidae, Ariidae, Carangidae, Gerreidae, Clupeidae e Paralichthyidae na área de estudo é apoiada por Szpilman (1994). Segundo este autor, a costa sul do Brasil é responsável por um grande volume de atividade pesqueira no país, sendo as espécies mais importantes pertencentes às famílias Sciaenidae, Ariidae, Clupeidae e Paralichthyidae.

A maior riqueza de espécies por família foi encontrada para Sciaenidae, com 8 espécies. Esta família, tem sido referida, em vários trabalhos, como a mais representativa em riqueza de espécies e abundância numérica para ambientes estuarinos, apresentando alta atividade reprodutiva para estes ambientes e áreas adjacentes (Travassos \& Paiva, 1957; IsaacNahum \& Vazzoler, 1987). Sciaenidae, com ampla distribuição geográfica e elevado número de espécies, integram a ictiofauna de cinco continentes, representando uma das famílias mais expressivas da atualidade (Chao, 1978). Embora registrando a sua ocorrência para águas mais profundas, a maioria das espécies habita águas costeiras rasas, próximas de estuários, constituindo um dos mais importantes recursos pesqueiros do Atlântico e grande relevância para o homem como fonte alimentar (Menezes \& Figueiredo, 1980).

Para Sciaenidae, as espécies que mais se destacaram em número de exemplares foram Stellifer rastrifer $(\mathrm{N}=605)$ e Micropogonias furnieri $(\mathrm{N}=247)$. Esta última espécie, vulgarmente conhecida por corvina, tem uma grande representatividade na produção pesqueira do estado de Santa Catarina, principalmente quando considerada a pesca artesanal praticada em ambientes de manguezais. Segundo Silva 
HOSTIM-SILVA, M. et al:: Peixes do rio Itajaí-açu.

Tabela II. - Lista das espécies identificadas e respectivas informações quantitativas: Abundância numérica ( $N$ ); amplitudes de comprimento total (CT) e categoria de frequência em que se incluem ( $\mathrm{MF}=$ muito frequentes; $F=$ frequente; $\mathrm{PF}=$ pouco frequente e $\mathrm{O}=$ ocasional).

\begin{tabular}{|c|c|c|c|}
\hline Espécies & $\begin{array}{l}\text { Abundância numérica } \\
\text { (N) }\end{array}$ & $\begin{array}{c}\text { CT } \\
\text { Mín - Max }\end{array}$ & Categoria \\
\hline Anchoa spinifer & 6 & $120 \quad 145$ & $\mathrm{O}$ \\
\hline Lycengraulis grossidens & 17 & $58 \quad 203$ & PF \\
\hline Cetengraulis edentulis & 2 & 98 & $\mathrm{O}$ \\
\hline Harengula clupeola & 7 & $\begin{array}{ll}40 & 107\end{array}$ & $\mathrm{O}$ \\
\hline Cathorops spixii & 106 & $\begin{array}{ll}61 & 263\end{array}$ & PF \\
\hline Genidens genidens & 612 & $60 \quad 388$ & $\mathrm{~F}$ \\
\hline Netuma barba & 89 & $73 \quad 460$ & $\mathrm{~F}$ \\
\hline Pimelodus maculatus & 2 & $195 \quad 275$ & $\mathrm{O}$ \\
\hline Mugil curema & 4 & $140 \quad 250$ & $\mathrm{O}$ \\
\hline Centropomus parallelus & 2 & $236 \quad 259$ & $\mathrm{O}$ \\
\hline Selene setapinnis & 3 & 76 & $\mathrm{O}$ \\
\hline Selene vomer & 3 & $48 \quad 52$ & $\mathrm{O}$ \\
\hline Trachinotus falcatus & 4 & 58 & $\mathrm{O}$ \\
\hline Diapterus rhombeus & 9 & $90 \quad 125$ & $\mathrm{PF}$ \\
\hline Eucinostomus melanopterus & 45 & 214 & $\mathrm{PF}$ \\
\hline Eucinostomus gula & 8 & $113 \quad 133$ & $\mathrm{O}$ \\
\hline Eugerres brasilianus & 1 & 155 & $\mathrm{O}$ \\
\hline Pomadasys corvinaeformis & 16 & $70 \quad 130$ & $\mathrm{O}$ \\
\hline Bairdiella ronchus & 38 & 270 & MF \\
\hline Cynoscion leiarchus & 07 & $122 \quad 200$ & $\mathrm{O}$ \\
\hline Isopisthus parvipinnis & 1 & 240 & $\mathrm{O}$ \\
\hline Micropogonias furnieri & 247 & $14 \quad 236$ & MF \\
\hline Ophioscion punctatissimus & 12 & $80 \quad 167$ & $\mathrm{O}$ \\
\hline Paralonchurus brasiliensis & 11 & $35 \quad 223$ & $\mathrm{PF}$ \\
\hline Stellifer brasiliensis & 4 & 64 & $\mathrm{O}$ \\
\hline Stellifer rastrifer & 605 & $20 \quad 225$ & $\mathrm{~F}$ \\
\hline Awaous tajasica & 4 & 132 & $\mathrm{O}$ \\
\hline Bathygobius soporator & 3 & $\begin{array}{ll}79 & 112\end{array}$ & $\mathrm{O}$ \\
\hline Gobioides braussonnetii & 2 & $110 \quad 140$ & $\mathrm{O}$ \\
\hline Gobionellus oceanicus & 4 & $95 \quad 150$ & $\mathrm{PF}$ \\
\hline Chaetodipterus faber & 4 & $65 \quad 120$ & $\mathrm{PF}$ \\
\hline Trichiurus lepturus & 2 & $370 \quad 1140$ & $\mathrm{O}$ \\
\hline Catathyridium garmani & 8 & 177 & PF \\
\hline Citharichthys spilopterus & 94 & 120 & MF \\
\hline Symphurus tessellatus & 110 & 173 & MF \\
\hline Sphoeroides testudineus & 11 & 60 & $\mathrm{O}$ \\
\hline Lagocephalus laeviagatus & 110 & 141 & $\mathrm{PF}$ \\
\hline Cyclichthys spinosus & 4 & 30 & $\mathrm{PF}$ \\
\hline
\end{tabular}


(1982), essa espécie pode ser caracterizada por desovar na região oceânica ou em águas de salinidade elevada, passando no estágio larval ou juvenil para o estuário para se desenvolver.

Foi assinalada a presença de Lycengraulis grossidens, Cathorops spixii, Genidens genidens, Netuma barba, Micropogonias furnieri, Stellifer rastrifer, Citharichthys spilopterus e Symphurus tessellatus, durante todo o ciclo anual. Contrariamente, algumas espécies da família Sciaenidae como Cynoscion leiarchus, Isophisthus parvipinnis, Ophioscion punctatissimus e Stellifer brasiliensis só marcaram presença nos trimestres referentes ao outono e inverno. Este fato pode estar relacionado com alterações das condições hidrodinâmicas, hábitos alimentares e variações sazonais relacionadas com reprodução e recrutamento.

A espécie mais abundante nas amostragens foi Genidens genidens. A família Ariidae, domina a costa sudeste do Brasil, em comunidades de peixes de estuários com margens cobertas por manguezais (Clezar et al., 1994). Esta espécie dominou as áreas com maior influência marinha, apresentando as maiores abundâncias no trimestre correspondente ao inverno.

Com exceção das espécies Selene vomer e S. setapinnis, os exemplares restantes da família Carangidae só marcaram presença no outono e inverno. Deste modo, as espécies acima referidas poderão ser consideradas espécies acidentais, sendo que a sua captura no interior do estuário não resulta da existência de uma população residente ou da migração sazonal de uma população costeira, mas de circunstâncias excepcionais de caráter totalmente aleatório.

Em todo o ciclo anual apenas foi capturada uma espécie de água doce (Pimelodus maculatus) na área mais a montante do rio. De acordo com Yáñez-Arancibia \& Nugent (1977), as comunidades variam a composição e abundância de espécies de acordo com as condições hidrológicas do sistema, em conseqüência das estações do ano e a localização dentro do estuário bem como os seus gradientes de salinidade.

As coletas demonstraram a existência, na área de estudo, de várias espécies de peixes de valor comercial, tais como pescadas, linguados, bagres e corvinas. Esta informação torna-se relevante para comprovar a importância sócio-econômica da área como local de atividade pesqueira para as comunidades ribeirinhas e mesmo para toda a região do Vale do Itajaí.

Todas as famílias foram representadas por um grande número de indivíduos juvenis, o que demonstra a importância da foz do rio como ambiente de crescimento. Esta constatação vem enfatizar as observações realizadas para outras regiões estuarinas, reforçando que estes ambientes servem de abrigo para peixes de pequeno porte (Chao, 1982; Rodrigues et al., 1994; Chaves \& Pichler, 2000). A grande abundância de indivíduos jovens de todas as espécies no Rio Itajaí - Açú, vem ainda reforçar as atribuições sobre a sua função de criadouro natural e a importância da sua preservação.

\section{AGRADECIMENTOS}

Os autores agradecem toda a equipe do laboratório de Ciências Ambientais do CTTMarUNIVALI pelo auxílio nos trabalhos de campo e de laboratório, em especial aos colegas e amigos: Norberto Ghizoni; Aluízio Caffé, Fábio Lopes; Leonardo Machado; Áthila Bertoncini Andrade. Muito obrigado pelo estímulo e o apoio em todas as longas horas de trabalho e aprendizado. Nosso muito obrigado aos revisores, que muito contribuíram com suas críticas e importantes sugestões. 


\section{REFERÊNCIAS BIBLIOGRÁFICAS}

Amanieu, M. \& G. Lasserre. 1982. Organisation et évolution des peuplements lagunaires. Oceanologica Acta. International Symposium on coastal lagoons, SCOR/ IABO/UNESCO, Bordeaux, France, 8-14 Septembre, 1981 SP: 201-213.

Barletta, M. \& M.F. M. Corrêa. 1992. Guia para identificação de peixes da costa do Brasil. Editora UFPR, Curitiba, PR, 121p.

Barnes, R. S. K. 1980. Coastal lagoons. Cambridge University Press, Cambridge. $106 \mathrm{p}$.

Chao, L. N. 1978. Sciaenidae. In: Fisher, W. (ed) FAO species identification sheets for fishery purpose. Western Central Atlantic (fishing area 31). Rome, FAO. pag. var.

Chao, L. N., Pereira, L. E., Viera, J. P., Benvenuti, M. A. \& L. P. R. Cunha. 1982. Relação Preliminar dos Peixes Estuarinos e Marinhos da Lagoa dos Patos e Região Costeira Adjacente, Rio Grande do Sul, Brasil, Atlântica, 5 (1): 67-75.

Chaves, P.T.C. \& H. A. Pichler. 2000. Variações espaço-temporais na atividade alimentar de peixes num ambiente estuarino (Baía de Guaratuba, Paraná, Brasil). Acta Biológica Leopoldensia, 22(2):277-287.

Chaves, P.T. \& A. L. Vendel. 2001. Nota complementar sobre a composição ictiofaunística da Baía de Guaratuba, Paraná, Brasil. Revta brás. Zool. 18(Supl. 1):349-352.

Clezar, L.; Ribeiro, G. C. \& M. Hostim-Silva. 1994. Relação peso total/comprimento total e factor de condição da manjuba Cetengraulis edentulus (CUVIER, 1828) (Clupeiformes - Engraulididae) no Manguezal do Itacorubi, SC, Brasil. Revista de Arqueologia, Biologia e Tecnologia, 37 (3): 685-691.

Daget, J. 1976. Les modèles mathématiques en écologie. Masson, Paris. $172 \mathrm{p}$.

Figueiredo, J. L. \& N. A. Menezes. 1978. Manual de peixes marinhos do sudeste do
Brasil. II - Teleostei (1). Museu de Zoologia da Universidade de São Paulo, 110 p.

Figueiredo, J. L. \& N. A. Menezes. 1980. Manual de peixes marinhos do sudeste do Brasil. III - Teleostei (2). Museu de Zoologia da Universidade de São Paulo, $90 \mathrm{p}$.

Fisher, W. 1978. Fao species identification sheets for fisheries porposes, Western Central Atlantic (Fishing Area 31). FAO, v. 1-7.

Isaac-Nahum, V. J. \& A. M. C. Vazzoler. 1987. Biologia reprodutiva de Micropogonias furnieri (Desmarest, 1823) (Teleostei, Sciaenidae). relação gonadossomática, comprimento e peso dos ovários como indicadores do período de desova. Bol. Inst. oceanogr. São Paulo, 35 (2): 123-134.

Menezes, N. A. \& J. L. Figueiredo. 1980. Manual de peixes marinhos do Sudeste do Brasil. IV Teleostei (3). São Paulo, Museu de Zoologia, USP, $98 \mathrm{p}$.

Menezes, N. A. \& J. L. Figueiredo. 1985. Manual de peixes marinhos do Sudeste do Brasil. V Teleostei (4). São Paulo, Museu de Zoologia, USP, $105 \mathrm{p}$.

Moyele, P. B. \& J. J. Cech. 1988. Fishes: An introduction to icthyology. $2^{\text {nd }} \mathrm{ed}$. Prentece Hall, Englewood Cliffs, New Jersey, 559 p.

Nelson, J.S. 1994. Fishes of the world. 3rd edition, John Wiley \& Sons, New York, 600p.

Potter, I. C.; Beckley, L. E.; Whitfield, A. K. \& R. C. J. Lenanton. 1990. Comparisons between the roles played by estuaries in the life cycles of fishes in temperate western Australia and southern Africa. Environmental Biology of Fishes, 28: 143178.

Rodrigues, A. M. T.; Pereira, M. T.; Wegner, P. Z.; Branco, J. O.; Clezar, L; Hostim-Silva, M. \& E. Soriano-Sierra. 1994. Manguezal do Rio Camboriú: Presevação e Controle da Qualidade Ambiental. Coleções Meio Ambiente, Série Estudos de Pesca, IBAMA, 13: 1-63. 
Silva, C. P. 1982. Ocorrência, distribuição e abundância de peixes na região estuarina de Tramandaí, Rio Grande do Sul. Atlântica, 5: 49-66.

Soriano-Sierra, E. \& B. Sierra De Ledo.(ed). (1998). Ecologia e gerenciamento do manguezal de Itacorubi. FEPEMA N 003, Florianópolis, SC.408p.

Szpilman, M. 1994. A practical guide to the identification of Brazilien Coastal fishes. Aqualung guide to fishes, SP, Brasil. 325p.

Travassos \& M. P. Paiva. 1957. Lista dos Sciaenidae Marinhos Brasileiros, Contendo Chave de Classificação e Proposta de "nomes vulgares oficiais". Bol. Inst. Oceanog. São Paulo, 8: 139-169.
Yáñez-Arancibia, A. \& R. S. Nugent. 1977. El papel ecológico de los peces en estuario y lagunas costeras. Anais del Instituto de ciencias del Mar y Limnologia de la Universidad Nacional Autónoma de México, 4 ( 1 ): 107-113.

Yáñez-Arancibia, A.; Lara-Domínguez, A. L., Chavance, P. \& D.F. Hernández. 1983. Environmental behavior of Terminos Lagoon ecological system, Campeche, México. Anais del Instituto de ciencias del Mar y Limnologia de la Universidad Nacional Autónoma de México 10 (1):137-176. 